\title{
Temporomandibular dislocation caused by pancreatic cancer metastasis: A case report
}

\author{
SHINPEI MATSUDA ${ }^{1}$, HITOSHI YOSHIMURA ${ }^{1}$, SADAHIKO KONDO $^{2}$ and KAZUO SANO ${ }^{1}$ \\ ${ }^{1}$ Department of Dentistry and Oral Surgery, Unit of Sensory and Locomotor Medicine, Division of Medicine, \\ Faculty of Medical Sciences, University of Fukui, Fukui 910-1193; ${ }^{2}$ Department of Oral \\ and Maxillofacial Surgery, Fukui Prefectural Hospital, Fukui 910-8526, Japan
}

Received March 22, 2017; Accepted July 27, 2017

DOI: 10.3892/ol.2017.6951

\begin{abstract}
Metastasis-induced dislocation of the temporomandibular joint (TMJ) is rare. The present study describes a case of TMJ dislocation caused by metastasis from pancreatic cancer, and discusses this in the context of literature on occlusal abnormality and/or dislocation due to metastasis. In the present case, unilateral TMJ dislocation was suspected when the patient first presented; destructive bone changes were not observed on conventional radiographs, and magnetic resonance imaging (MRI) revealed a tumorous lesion. The present case suggests that healthcare professionals should consider whether a malignant disease is present in cases of occlusal abnormality and/or dislocation of the TMJ, and that, in patients with TMJ dislocation that cannot easily be repositioned, additional imaging examinations, including MRI, should be performed as soon as possible, regardless of whether destructive bone changes are present. Metastasis to the TMJ is typically associated with generalized skeletal metastasis in the final stage of malignancy. Therefore, it is crucial to consider the possibility of cervical spine metastasis in order to decrease the risk of cervical fracture when attempting reduction of a TMJ dislocation, particularly in patients with a history of cancer.
\end{abstract}

\section{Introduction}

Dislocation of the temporomandibular joint (TMJ) is defined as an excessive forward movement of the condyle beyond the articular eminence with complete separation of the articular surfaces and fixation in that position (1). Although dislocation or subdislocation of the TMJ is typically bilateral, it may be unilateral and cause occlusal abnormality (2). TMJ dislocation

Correspondence to: Dr Shinpei Matsuda, Department of Dentistry and Oral Surgery, Unit of Sensory and Locomotor Medicine, Division of Medicine, Faculty of Medical Sciences, University of Fukui, 23-3 Matsuokashimoaizuki, Eiheiji-cho, Yoshida-gun, Fukui 910-1193, Japan

E-mail: shinpeim@u-fukui.ac.jp

Key words: temporomandibular dislocation, occlusal abnormality, pancreatic cancer, metastasis, tumor marker is typically associated with hypermobility of the mandible and poor development of the articular fossa $(1,2)$. The differential diagnosis for diseases leading to malocclusion and/or dislocation in this region includes dental malocclusion without skeletal anomalies, skeletal malocclusion, articular disc displacement, thickness of the retrodiscal tissue, trauma, infection, arthritis, tumor or tumor-like lesions, and neuromuscular diseases $(3,4)$. TMJ dislocation is rarely associated with tumor or tumor-like lesions, and cases of metastasis-induced TMJ dislocation are extremely rare (5).

The 5-year survival rate of pancreatic cancer (PC) was reported to be no more than $5 \%$ in China (6) and the USA (7). Patients with PC often exhibit no symptoms in the early stage, and the early clinical symptoms of PC that are present are typically non-specific, including back and shoulder pain, dyspepsia, dysphagia, changes in bowel habits and lethargy (8). Therefore, patients with PC are often diagnosed at an advanced stage and distant metastases are occasionally detected at the initial diagnosis (8). The most frequent metastatic sites are the liver and peritoneum, followed by the lungs (9). PC rarely metastasizes to the head and neck region, and there are few reports in the English literature of PC metastasizing to the mandibular condyle (10).

The current report presents a case of TMJ dislocation caused by PC metastasis, and discusses this in the context of literature on occlusal abnormality and/or TMJ dislocation due to metastasis.

\section{Case report}

An 85-year-old female was referred to the Department of Dentistry and Oral Surgery, the University of Fukui Hospital (Fukui, Japan) in January 2012 presenting with trismus, mandibular deviation toward the left side, and spontaneous pain in the right auricular and buccal region for 3 months. Physical examination revealed trismus and facial asymmetry due to mandibular deviation toward the left side and right preauricular depression. Panoramic radiography and lateral oblique transcranial projection revealed anterior displacement of the right mandibular condyle, whereas the left condyle was located in the mandibular fossa (Figs. 1 and 2). The patient was diagnosed with right temporomandibular dislocation upon presentation to the Department of Dentistry and Oral Surgery, the University of Fukui Hospital, and reduction of the dislocation was 


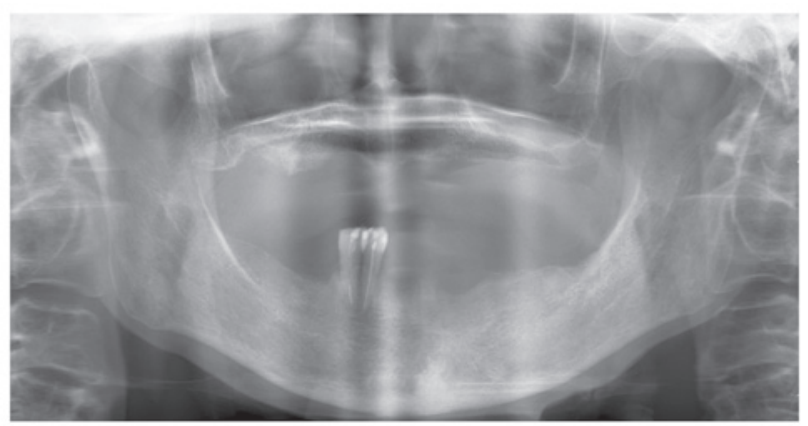

Figure 1. Panoramic radiograph revealing anterior deviation of the right mandibular condyle.

unsuccessfully attempted. Magnetic resonance imaging (MRI) revealed a tumorous lesion extending from the right temporal muscle to the right sphenoid bone, right temporomandibular dislocation and anterior displacement of the right articular disc (Fig. 3). Enhanced computed tomography (CT) revealed a tumorous lesion from the right temporal muscle to the right pterygoid muscles, and intracranial and orbital extension was observed (Fig. 4). Three-dimensional CT using the OsiriX program (version 4.1.2; Pixmeo SARL, Geneva, Switzerland) demonstrated dislocation of the right TMJ (Fig. 5) (11). Technetium-99 m methylene diphosphonate and gallium-67 citrate scintigraphy showed abnormally increased uptake in the right temporal and sphenoidal regions and multiple sites throughout the body, including the skull bones, upper and lower jaw, vertebrae, ribs, scapula, sternum and pelvic bone, and in each femur (Fig. 6). An enhanced CT scan of the chest and abdomen revealed a $4-\mathrm{cm}$ nodule in the tail of the pancreas and a hypoenhanced mass in the liver (Fig. 7). Laboratory examination revealed increased C-reactive protein $(0.89 \mathrm{mg} / \mathrm{dl}$; normal range, $<0.32 \mathrm{mg} / \mathrm{dl}$ ), carcinoembryonic antigen (CEA; $83.5 \mathrm{ng} / \mathrm{ml}$; normal range, $<5.0 \mathrm{ng} / \mathrm{ml})$, carbohydrate antigen 19-9 (CA19-9; 52,541.7 U/ml; normal range, <37.0 U/ml) and DUPAN-2 (2,660 U/ml; normal range, 0-150 U/ml), which are tumor markers of gastrointestinal cancer and PC. Therefore, the patient was diagnosed with PC with multiple metastases by a gastroenterologist in the Department of Gastroenterology, the University of Fukui Hospital. In addition, the patient was diagnosed with PC metastasis-associated dislocation of the TMJ. The patient and the patient's family chose not to undergo further examinations, including biopsy. Chemotherapy and palliative therapy were proposed instead of surgery due to the age and general condition of the patient and the progression of the lesion. The patient opted for palliative therapy and was discharged from hospital and succumbed to cachexia 2 months after initially presenting with disease.

Written informed consent for the publication of this case report and associated images was obtained from the patient's family.

\section{Discussion}

Kolokythas et al (10) reported in their review article that metastasis to the oral cavity is rare in PC, with an incidence of $1-8 \%$, and metastasis to the TMJ is extremely rare. Approximately $33 \%$ of cases of previously undiscovered primary tumor

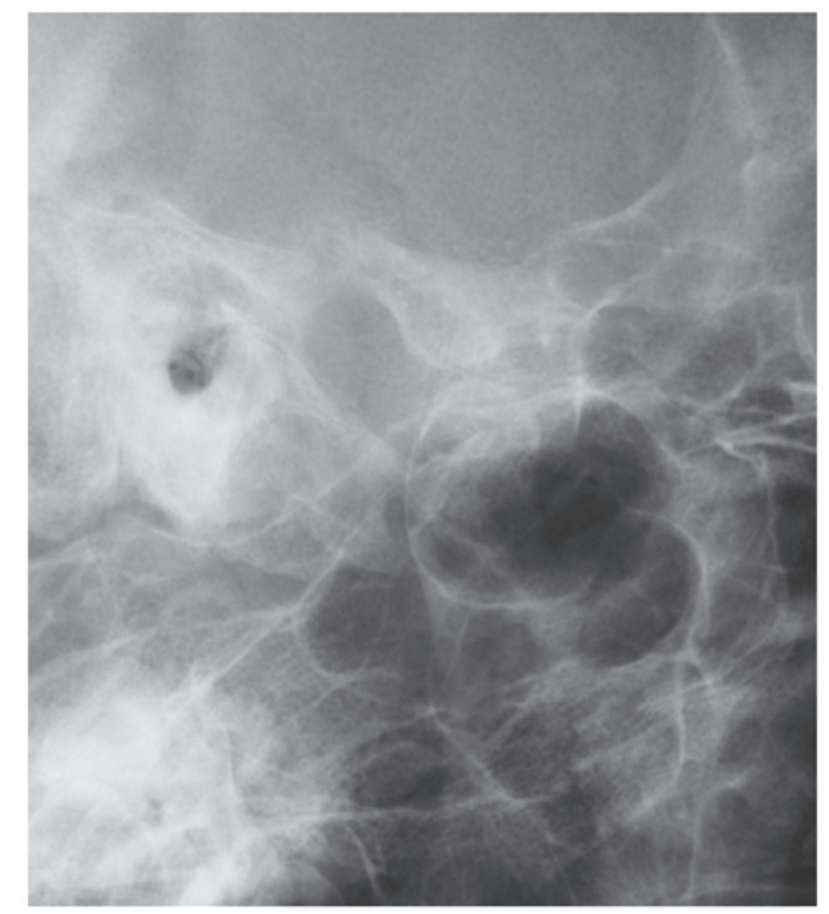

Figure 2. Lateral oblique transcranial projection radiograph revealing frontal deviation of the right mandibular condyle.

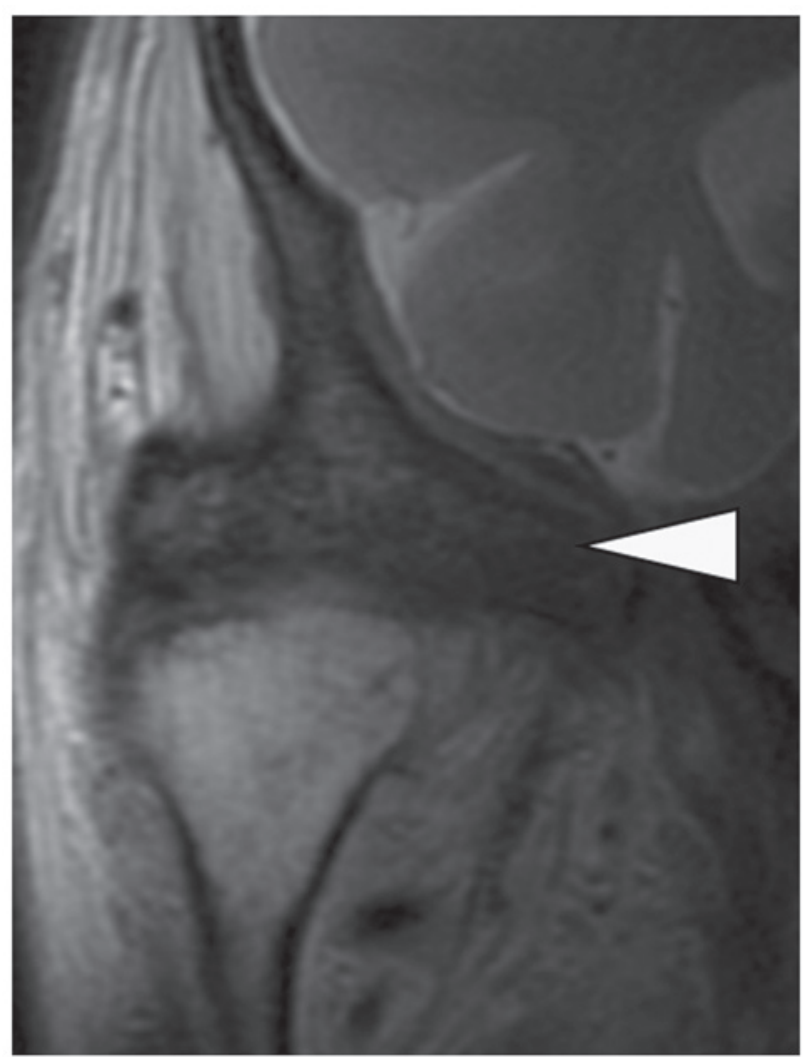

Figure 3. T2-weighted magnetic resonance imaging scan revealing a tumorous lesion (white arrowhead) from the right temporal bone to the right sphenoid bone.

presented with oral metastasis as the first indication (10). According to the literature on 796 cases of oral and maxillofacial metastases, the most common metastatic site was the 


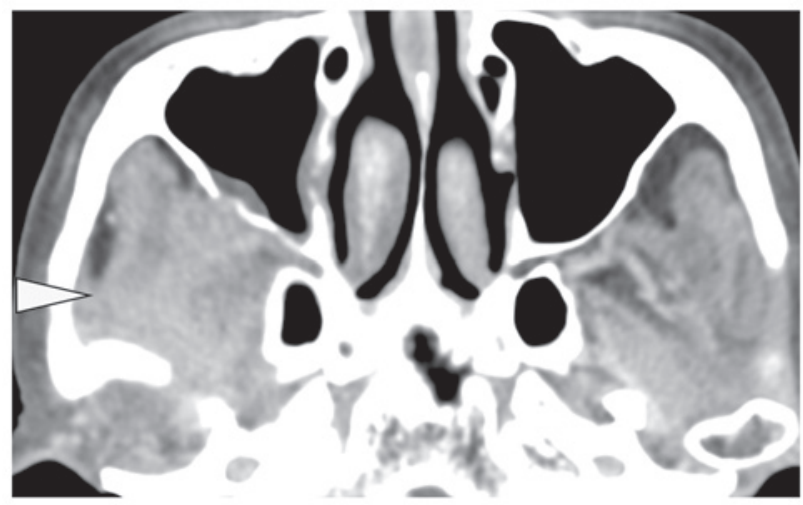

Figure 4. Head and neck enhanced computed tomography scan revealing a tumorous lesion (white arrowhead) of $\sim 3 \mathrm{~cm}$ at the maximum diameter, extending from the right temporal muscle to the right pterygoid muscles with intracranial and orbital extension, with internal necrosis or degeneration. The right condyle was not observed due to anterior displacement induced by the tumor. The left condyle was located in the mandibular fossa

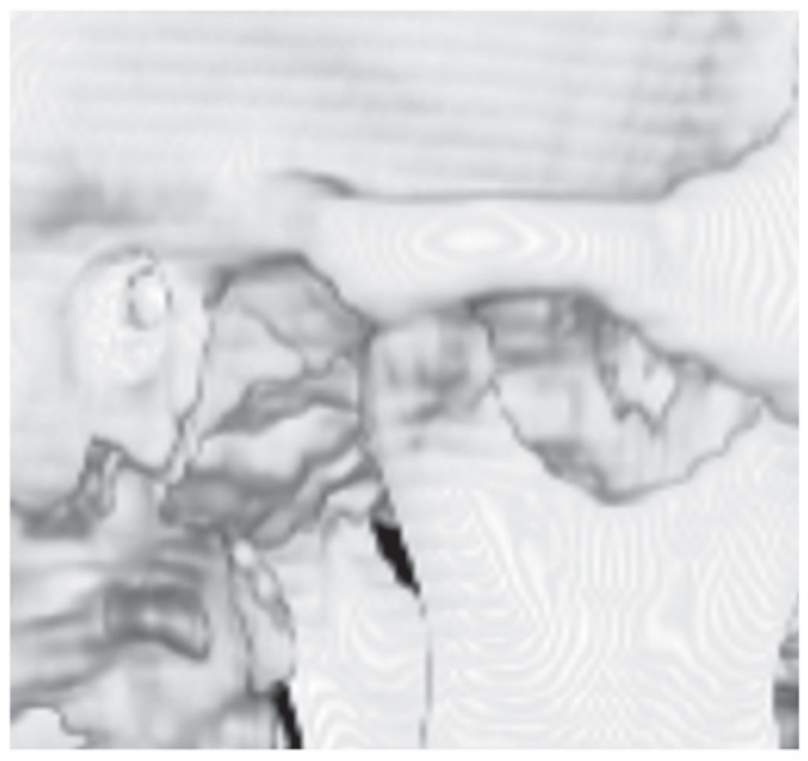

Figure 5. Three-dimensional computed tomography scan using the OsiriX program, demonstrating dislocation of the right temporomandibular joint.

mandible (283/796 cases, $35.6 \%$ ), and only 39/796 cases $(4.9 \%)$ were of metastasis to the mandibular condyle (12). A review of the literature from between 1954 and 2013 identified 66 cases of metastasis to the TMJ (29 males, 35 females and 2 of unknown gender; age range, 32-85 years; mean age \pm standard deviation, $59.0 \pm 11.4$ years); pain and/or swelling were common clinical symptoms in these patients (13).

Rubin et al (14) reported that symptoms of TMJ dysfunction, including pain, trismus and mandibular deviation, coupled with swelling and radiographic evidence of a destructive lesion or pathological fracture, may suggest the presence of a malignancy, either primary or metastatic. A tumor occurring in the TMJ often obstructs the opening of the jaws; however, in the present study, the tumor in the mandibular fossa caused TMJ dislocation by pushing the condyle out of the fossa (3). The literature review revealed 11 cases of occlusal abnormality and/or TMJ dislocation due to metastasis (5 males and 6 females; age range,

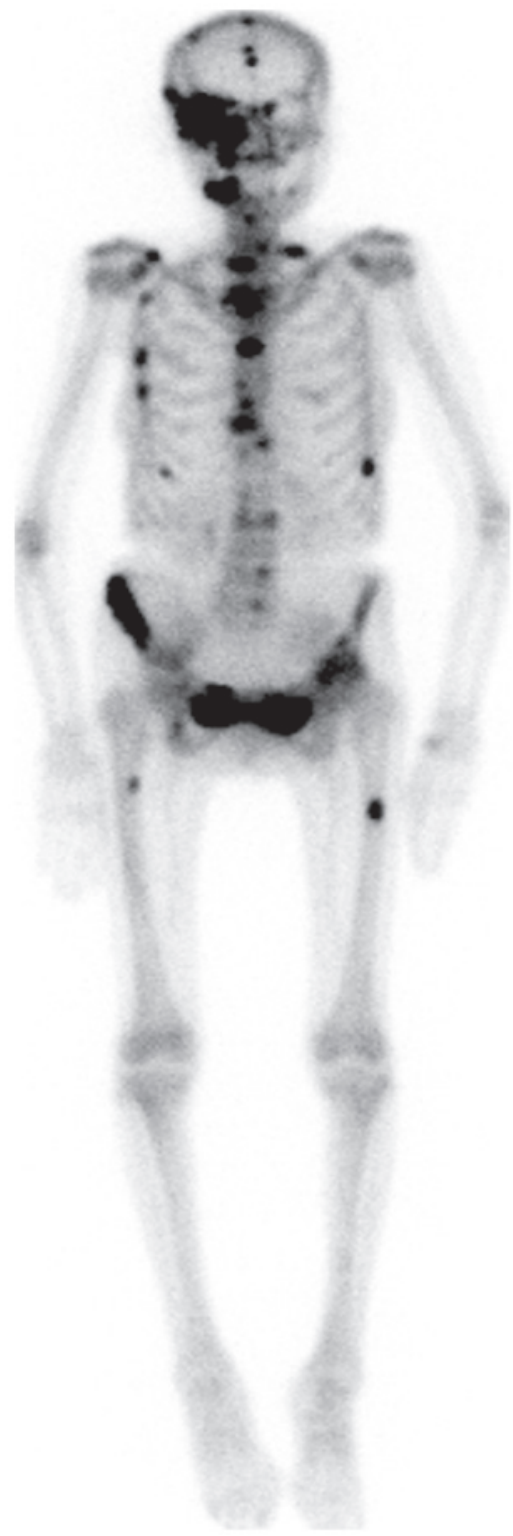

Figure 6.99mTc scintigraphy exhibiting abnormally increased MDP uptake in the right temporal and sphenoidal region and multiple throughout the body, including the skull, upper and lower jaw, vertebrae, ribs, scapula, sternum, pelvic bone and each femur.

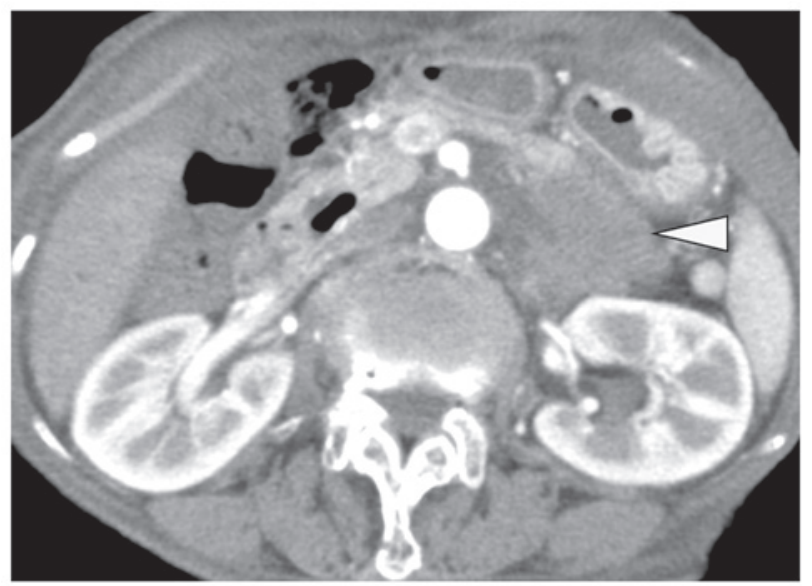

Figure 7. Abdominal enhanced computed tomography scan revealing a poorly enhanced tumor ( $4 \mathrm{~cm}$ at the maximum diameter, white arrowhead) in the pancreatic tail. 


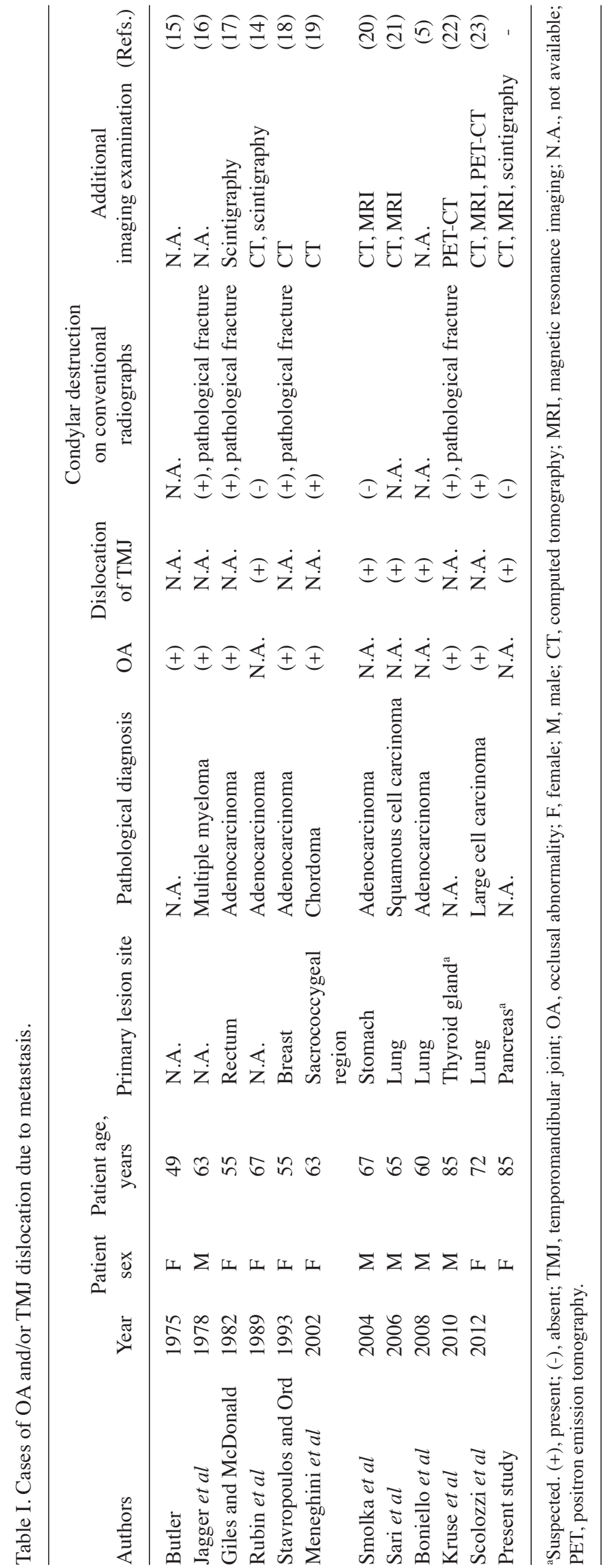


49-85 years; mean age \pm standard deviation, 63.7 \pm 9.6 years), 4 of which were cases of TMJ dislocation (Table I) $(5,14-23)$. Condylar destruction, including pathological fracture, was often observed using conventional radiography in cases of occlusal abnormality, whereas it was not observed in cases of TMJ dislocation (16-18,22). Smolka et al (20) reported a case of TMJ dislocation due to synovial metastasis without destructive bone changes, in which a tumorous lesion was revealed using MRI. In the present study, unilateral TMJ dislocation was suspected when the patient initially presented, since destructive bone changes were not observed using conventional radiography and MRI revealed a tumorous lesion. The present study highlights the importance of considering whether a malignant disease is present in cases of occlusal abnormality and/or dislocation of the TMJ, and suggests that, in patients with TMJ dislocation that cannot easily be repositioned, additional imaging examinations, including MRI, should be performed as soon as possible, regardless of whether destructive bone changes are observed.

In cases of metastasis to the TMJ, the most common primary site was the breast and the most common histological type of tumor was adenocarcinoma (13). Metastasis to the TMJ was typically associated with generalized skeletal metastasis in the final stage of malignancy, and mortality occurred in the majority of patients within 1 year after the diagnosis of metastasis (24). The mechanism underlying metastasis to the mandible is considered to be hematogenous spread, since there is no lymphatic system in the jaw bone $(10,24)$. Metastases are more likely to occur in regions with increased red marrow concentration since malignant cells become lodged in red marrow as the initial site for skeletal metastasis, or occur in regions with abrupt angulation of the vessel due to the decelerated blood flow, which aids the deposition of malignant cells $(25,26)$. In previous studies, the majority of mandibular metastases (53\%) occurred in the molar and premolar regions where red marrow was present and blood flow was decelerated $(12,26)$. By contrast, metastasis to the mandibular condyle rarely occurs due to a lack of substantial hematopoietic marrow and limited local blood supply (10). In addition, synovial metastases, among which the knee is the most common site, appear to be rare (27). The normal synovium contains blood and lymphatic vessels. Therefore, this tissue could be associated with metastasis (27). However, the majority of synovial metastases are reportedly the direct extension of bone metastases into the adjacent joint, while hematogenous metastasis is rare (27). McConnell et al (27) reported that synovial metastases occurred following knee arthroplasty in 2 patients with gastrointestinal cancer. Hematogenous metastasis to the mandible and synovial membrane may occur through the Batson venous plexus (28).

CEA, CA19-9 and DUPAN-2 are recognized markers for PC $(29,30)$. Ni et al (29) reported that the sensitivity of CA19-9 alone was $80 \%$, and that the specificity of CEA combined with CA19-9 was $84 \%$ in the diagnosis of PC. Furthermore, the median survival time of patients with a CA19-9 serum level $\geq 1,000 \mathrm{U} / \mathrm{ml}$ was 6 months (29). Goonetilleke and Siriwardena (30) reported that the sensitivity and specificity of CA19-9 for diagnosing PC were 79 and 82\%, respectively, and those of DUPAN-2 were 65 and $80 \%$, respectively. Furthermore, a CA19-9 serum level $>40 \mathrm{U} / \mathrm{ml}$ with positive or equivocal radiological findings was highly suggestive of a diagnosis of PC (30). In the present study, the serum levels of tumor markers specific for PC (CEA, CA19-9 and DUPAN-2) were increased beyond the normal range. These results were consistent with those of the imaging examinations, and suggested that PC was present.

To conclude, the present study suggested that healthcare professionals should consider whether a malignancy is present in cases of occlusal abnormality and/or TMJ dislocation, and that, in patients with TMJ dislocation that cannot easily be repositioned, additional imaging examinations, including MRI, should be performed as soon as possible, regardless of whether there are destructive bone changes. Metastasis to the TMJ is typically associated with generalized skeletal metastasis in the final stage of malignancy (24); in the present case, destructive changes to the cervical spine due to metastasis was observed on the CT scan shortly before the patient succumbed to the disease. Therefore, it is necessary to consider the possibility of cervical spine metastasis in order to decrease the risk of cervical fracture when attempting reduction of a TMJ dislocation, particularly in patients with a history of cancer.

\section{References}

1. Vasconcelos BC and Porto GG: Treatment of chronic mandibular dislocations: A comparison between eminectomy and miniplates. J Oral Maxillofac Surg 67: 2599-2604, 2009.

2. Cardoso AB, Vasconcelos BC and Oliveira DM: Comparative study of eminectomy and use of bone miniplate in the articular eminence for the treatment of recurrent temporomandibular joint dislocation. Braz J Otorhinolaryngol 71: 32-37, 2005.

3. Brustowicz KA and Padwa BL: Malocclusion in children caused by temporomandibular joint effusion. Int J Oral Maxillofac Surg 42: 1034-1063, 2013.

4. Hasegawa T, Shibuya Y, Minamikawa T and Komori T: Two cases of posterior open bite caused by the thickness of retrodiscal tissue in the temporomandibular joint. Int J Oral Maxillofac Surg 43: 1104-1107, 2014.

5. Boniello R, Gasparini G, D'Amato G, Di Petrillo A and Pelo S: TMJ metastasis: A unusual case report. Head Face Med 4: 8 , 2008.

6. Luo J, Xiao L, Wu C, Zheng Y and Zhao N: The incidence and survival rate of population-based pancreatic cancer patients: Shanghai Cancer Registry 2004-2009. PLoS One 8: e76052, 2013.

7. Jemal A, Siegel R, Ward E, Hao Y, Xu J and Thun MJ: Cancer statistics, 2009. CA Cancer J Clin 59: 225-249, 2009.

8. Keane MG, Horsfall L, Rait G and Pereira SP: A case-control study comparing the incidence of early symptoms in pancreatic and biliary tract cancer. BMJ Open 4: e005720, 2014.

9. Rao R, Sadashiv SK, Goday S and Monga D: An extremely rare case of pancreatic cancer presenting with leptomeningeal carcinomatosis and synchronous intraparenchymal brain metastasis. Gastrointest Cancer Res 6: 90-92, 2013.

10. Kolokythas A, Miloro MB, Olsson AB and Miloro M: Metastatic pancreatic adenocarcinoma to the mandibular condyle: A rare clinical presentation. J Oral Maxillofac Surg 72: 83-88, 2014.

11. Osman R and Antoine R: Open-source software in medical imaging: Development of OsiriX. Int J CARS 1: 187-196, 2006.

12. Shen ML, Kang J, Wen YL, Ying WM, Yi J, Hua CG, Tang XF and Wen YM: Metastatic tumors to the oral and maxillofacial region: A retrospective study of 19 cases in West China and review of the Chinese and English literature. J Oral Maxillofac Surg 67: 718-737, 2009.

13. Pretzl C, Lübbers HT, Grätz KW and Kruse AL: Metastases in the temporomandibular joint: A review from 1954 to 2013. Rare causes for temporomandibular disorders. Swiss Dent J 124: 1067-1083, 2014 (In French, German).

14. Rubin MM, Jui V and Cozzi GM: Metastatic carcinoma of the mandibular condyle presenting as temporomandibular syndrome. J Oral Maxillofac Surg 47: 507-510, 1989.

15. Butler JH: Myofascial pain dysfunction syndrome involving tumor metastasis. Case report. J Periodontol 46: 309-311, 1975.

16. Jagger RG, Helkimo $M$ and Carlsson GE: Multiple myeloma involving the temporomandibular joint: Report of case. J Oral Surg 36: 557-559, 1978. 
17. Giles DL and McDonald PJ: Pathologic fracture of mandibular condyle due to carcinoma of the rectum. Oral Surg Oral Med Oral Pathol 53: 247-249, 1982.

18. Stavropoulos MF and Ord RA: Lobular adenocarcinoma of breast metastatic to the mandibular condyle. Report of a case and review of the literature. Oral Surg Oral Med Oral Pathol 75: 575-578, 1993.

19. Meneghini F, Castellani A, Camelin N and Zanetti U: Metastatic chordoma to the mandibular condyle: An anterior surgical approach. J Oral Maxillofac Surg 60: 1489-1493, 2002.

20. Smolka W, Brekenfeld C, Büchel P and Iizuku T: Metastatic adenocarcinoma of the temporomandibular joint from the cardia of the stomach: A case report. Int J Oral Maxillofac Surg 33: 713-715, 2004

21. Sari M, Serin GM, Inanli S and Kaya H: Condylar metastasis involoving TMJ and TMJ dislocation presenting as the initial manifestation of squamous lung cancer. Oral Oncol Extra 42: 224-226, 2006.

22. Kruse AL, Luebbers HT, Obwegeser JA, Edelmann L and Graetz KW: Temporomandibular disorders associated with metastases to the temporomandibular joint: a review of the literature and 3 additional cases. Oral Surg Oral Med Oral Pathol Oral Radiol Endod 110: e21-e28, 2010.
23. Scolozzi P, Becker M and Lombardi T: Mandibular condylar metastasis mimicking acute internal derangement of the temporomandibular joint. J Can Dent Assoc 78: c77, 2012.

24. Zachariades N: Neoplasms metastatic to the mouth, jaws and surrounding tissues. J Craniomaxillofac Surg 17: 283-290, 1989.

25. Basu S and Alavi A: Bone marrow and not bone is the primary site for skeletal metastasis: Critical role of [18F]fluorodeoxyglucose positron emission tomography in this setting. J Clin Oncol 25: 1297, 2007.

26. Banerjee SC: Metastasis to the mandible. Oral Surg Oral Med Oral Pathol 23: 71-77, 1967.

27. McConnell M, Kumar R, Amini B, Lin PP and Wang WL: Calcified synovial metastasis in the knee from renal cell carcinoma: A case report. Skeletal Radiol 46: 123-127, 2017.

28. Batson OV: The function of the vertebral veins and their role in the spread of metastases. Ann Surg 112: 138-149, 1940.

29. Ni XG, Bai XF, Mao YL, Shao YF, Wu JX, Shan Y, Wang CF, Wang J, Tian YT, Liu Q, et al: The clinical value of serum CEA, CA19-9, and CA242 in the diagnosis and prognosis of pancreatic cancer. Eur J Surg Oncol 31: 164-169, 2005.

30. Goonetilleke KS and Siriwardena AK: Systematic review of carbohydrate antigen (CA 19-9) as a biochemical marker in the diagnosis of pancreatic cancer. Eur J Surg Oncol 33: 266-270, 2007. 